\title{
Mapping of drift surfaces in toroidal systems with chaotic magnetic fields
}

\author{
S. S. Abdullaev \\ Institut für Plasmaphysik, Forschungszentrum Jülich GmbH, EURATOM Association, \\ Trilateral Euregio Cluster, D-52425 Jülich, Germany
}

\author{
A. Wingen \\ Institut für Theoretische Physik, Heinrich-Heine-Universität Düsseldorf, D-40225 Düsseldorf, Germany \\ K. H. Spatschek ${ }^{\text {a) }}$ \\ Institut für Plasmaphysik, Forschungszentrum Jülich GmbH, EURATOM Association, \\ Trilateral Euregio Cluster, D-52425 Jülich, Germany and Institut für Theoretische Physik, \\ Heinrich-Heine-Universität Düsseldorf, D-40225 Düsseldorf, Germany
}

(Received 15 December 2005; accepted 10 February 2006; published online 18 April 2006)

\begin{abstract}
Drift orbits of test particles are studied using a recently proposed Hamiltonian theory of guiding-center motion in toroidal systems. A symplectic mapping procedure in symmetric form is developed which allows a fast and accurate characterization of the Poincaré plots in poloidal cross sections. It is shown that the stochastic magnetic field acts differently on the onset of chaotic motion for co- and counterpassing particles, respectively. Resonant drift surfaces are shifted inward for the co-passing particles, and are shifted outward for the counterpassing particles, when compared with resonant magnetic surfaces. The overall result is an inward (outward) shift of chaotic zones of co-passing (counterpassing) particles with respect to the magnetic ergodic zone. The influence of a stationary radial electric field is discussed. It shifts the orbits farther inward for the co-passing particles and outward for the counterpassing particles, respectively. The shifts increase with the energies of the particles. A rotation of the magnetic field perturbations and its effect on drift motion is also investigated. Estimates for the local diffusion rates are presented. For applications, parameters of the dynamic ergodic divertor of the Torus Experiment for Technology-Oriented Research are used [Fusion Eng. Design 37, 337 (1997)]. (C) 2006 American Institute of Physics.
\end{abstract} [DOI: $10.1063 / 1.2181975$ ]

\section{INTRODUCTION}

Since the beginning of magnetic fusion research, the problem of particle and heat transport is in the focus of theoretical and experimental investigations. The reason for the strong interest in this problem lies in the unexpected large losses (anomalous transport). Although neoclassical (linear) transport theory was able to take care of the geometrical effects and large mean free paths (see, e.g., Ref. 1, and references therein), it was not able to resolve all the problems. As it is well known (see, for example, Ref. 2), in many cases the strong deviation of transport rates from classical predictions is due to nonlinear effects caused by (electrostatic as well as electromagnetic) fluctuations. In the past, many attempts have been made for a self-consistent theory of nonlinear transport; see, e.g., Refs. 3 and 4, and references therein.

For a better systematic understanding of anomalous transport in magnetically confined plasmas it was suggested in Ref. 5 to split the problem into two parts. One part deals with the development of fluctuations, and the other one considers the (passive) motion of (test) particles under the influence of the perturbations. Such separations are quite common in fluid turbulence where passive motion of scalars, vectors, particles, etc., has been investigated extensively. In fusion research there exists an additional, qualitatively im-

\footnotetext{
${ }^{a)}$ Electronic mail: spatschek@thphy.uni-duesseldorf.de
}

portant reason to investigate particle motion in given stochastic fields. Stochastic fields can be, and really are, also generated from outside (error fields or fields by purposely introduced specially designed external coils). Then, the enhanced transport of particles due to destruction of nested magnetic surfaces in the presence of nonaxisymmetric magnetic perturbations (see, e.g., Refs. 6-12) needs special consideration. An additional problem is the selective transport of particles in the presence of external static or rotating magnetic perturbations. This effect has been proposed as a mechanism for removal of helium ash and high energetic particles (see, e.g., Refs. 13-15).

In this paper, we discuss stochastic drift motion by using discrete maps. As is well known, magnetic field lines represent a continuous Hamiltonian system. The main goals of mapping models is to replace the original continuous dynamical system by a discrete iterative map which runs much faster then the small-step numerical integration (see Refs. 16-19). Mappings should be symplectic (or flux-preserving). They should have the same fixed points as the Poincaré map of the original system, and they should show the same regular and chaotic regions as the continuous evaluation. Many interesting mappings, especially for magnetic field lines, have been proposed in the past (see, for example, Refs. 19-23). The effect of the magnetic field perturbations on particle motion has attracted less attention, except, perhaps, Ref. 24. Although charged particles closely follow magnetic 
field lines, their orbits may deviate from the latter depending on the energy of the particles and the species. The deviations of electron orbits from the magnetic surfaces are much smaller than for ion orbits. Due to a toroidal drift the ion orbits are different for the co-passing, counterpassing, and trapped particles, respectively. Usually the perturbation field in toroidal systems has a strong radial dependence and is localized on the high-field side. In this work we intend to study in detail the possible effects of the perturbation fields on test ion orbits.

Equations for guiding-center motion in a Hamiltonian formulation are a convenient way to study the effect of chaotic magnetic fields on particle motion in collisionless plasmas. The Hamiltonian guiding-center equations in a magnetic coordinate system proposed in Refs. 25-28 allows, in principle, only a special form of the magnetic field that gives rise to chaotic field lines. The approach proposed in Ref. 29, and later generalized in Ref. 30 to time-dependent fields, allows one to consider more general forms of the magnetic field. But a practical application of the method may be complicated, because the method requires a coordinate system in which one of the covariant components of the magnetic $\mathbf{B}$ and the vector potential $\mathbf{A}$ simultaneously vanish, e.g., $A_{r}$ $=B_{r}=0$. Another Hamiltonian formulation of guiding-center motion in a toroidal system was recently proposed in Ref. 31 . It allows us to consider more general axisymmetric magnetic perturbations, including time-dependent ones. The idea of the method is close to the one proposed by Mynick in Refs. 32 and 33 to describe the guiding-center motion for large gyroexcursions in mirror and axisymmetric fieldreversed configurations. A six-dimensional Hamiltonian system of equations was obtained for drift motion in a toroidal coordinate system. The existence of an adiabatic invariant related with the radial gyro-oscillations allows one to reduce the six-dimensional Hamiltonian system to a fourdimensional one. In the present paper we shall further generalize that ansatz.

For quantitative predictions, we shall apply the theory to the dynamic ergodic divertor (DED) which has been installed at the Torus Experiment for Technology-Oriented Research (TEXTOR) (see Refs. 34 and 35). The DED introduces new features to the conventional concept of the ergodic divertor proposed in Refs. 36-42. Besides controlling the plasma edge by creating the resonant magnetic perturbations, the DED also permits the operation with a rotating magnetic field. The latter allows one to broaden the heat and the particle deposition on the divertor plates and to induce the plasma rotation as described in Ref. 43. The structure of the magnetic field in the DED operation has been extensively studied in the past in Refs. 20, 35, and 44-47.

In the present work we will investigate the effect of magnetic perturbations on a single test particle motion using the Hamiltonian approach mentioned above. Our attention will be concentrated on particle motion in a collisionless regime. It will reveal the effect of a pure magnetic field line stochasticity on the drift motion of particles without shadowing it with the effects of collisions and turbulence. The influence of an equilibrium electric field, as well as a rotating magnetic field, will be also considered. We shall mainly fo- cus on the shift of resonant drift surfaces from resonant magnetic surfaces and on the formation of a stochastic zone of drift motion. Radial diffusion rates of particles due to the chaotic magnetic fields will be also estimated.

The paper is organized as follows: The Hamiltonian equations for the guiding-center motion are presented in Sec. II within different formulations. The analysis of drift motion using Hamiltonian methods, i.e., action-angle variables and perturbation theory, are carried out in Sec. III. There we shall obtain formulas for the width of the drift islands, and we shall describe the mapping method to integrate Hamiltonian drift motion equations. The formation of chaotic zones, the effects of static electric and rotating magnetic fields are studied in Sec. IV. Radial diffusion coefficients of test particles are estimated in Sec. V. The obtained results are summarized in Sec. VI. In Appendix A, we present data for a realistic equilibrium magnetic field and the DED magnetic field perturbations to make quantitative estimates. Formulations in action-angle variables are commented on in Appendix B.

\section{HAMILTONIAN GUIDING-CENTER EQUATIONS}

We start with the Hamiltonian equations for the guidingcenter motion. The equations will be in a more general form than they were proposed in Ref. 31, i.e., without the $B_{\varphi}$ $\propto 1 / R$ assumption on the toroidal magnetic field.

We use a cylindrical coordinate system $(\hat{R}, \varphi, \hat{Z}$, where $\hat{R}$ is along the torus axis and $\varphi$ is the toroidal angle). Suppose that the magnetic field, including the perturbations, is completely determined by a vector potential $\mathbf{A}(\hat{R}, \varphi, \hat{Z}, \hat{t})$ $=\left(0, A_{\varphi}(\hat{R}, \varphi, \hat{Z}, \hat{t}), A_{Z}(\hat{R}, \varphi, \hat{Z}, \hat{t})\right)$. Because of a gauge invariance, the radial component $A_{R}$ can be always set to zero. The toroidal field is mainly determined by the $A_{Z}$ component, while the component $A_{\varphi}$ describes the mean poloidal field $\mathbf{B}_{p}$ and the magnetic perturbations $\mathbf{B}_{\text {pert }}: \mathbf{B}_{p}+\mathbf{B}_{\text {pert }}=\nabla \times \mathbf{e}_{\varphi} A_{\varphi}$. An electric field is incorporated via the potential $\Phi(\hat{R}, \varphi, \hat{Z}, \hat{t})$ which may contain an equilibrium potential $\Phi_{0}(\hat{R})$ as well as low-frequency electric fluctuations $\Phi_{1}(\hat{R}, \varphi, \hat{Z}, \hat{t})$. Let $R_{0}$ and $B_{0}$ be the major radius of the torus and the strength of the reference magnetic field, respectively. Introducing the normalized time $\omega_{c} \hat{t} \rightarrow t$, where $\omega_{c}=e B_{0} / m c$ is a reference gyrofrequency, the Hamiltonian equations for the particle motion can be written as

$$
\frac{d q_{i}}{d t}=\frac{\partial \mathcal{H}}{\partial p_{i}}, \quad \frac{d p_{i}}{d t}=-\frac{\partial \mathcal{H}}{\partial q_{i}}, \quad i=1,2,3
$$

where $\left(q_{1}, q_{2}, q_{3}\right)=\left(x=\left(\hat{R}-R_{0}\right) / R_{0}, \varphi, z=\hat{Z} / R_{0}\right)$ are the normalized coordinates, and $\left(p_{1}, p_{2}, p_{3}\right)=\left(p_{x}, p_{\varphi}, p_{z}\right)$ are the conjugate normalized canonical momenta. The Hamiltonian function $\mathcal{H}$ in (1) is

$$
\mathcal{H}\left(x, \varphi, z, p_{x}, p_{\varphi}, p_{z}, t\right)=\frac{1}{2} p_{x}^{2}+U\left(x ; \varphi, z, p_{\varphi}, p_{z}, t\right),
$$




$$
U=\frac{1}{2}\left[\frac{\left(p_{\varphi}-f_{\varphi}\right)^{2}}{(1+x)^{2}}+\left(p_{z}-f_{z}\right)^{2}\right]+\phi(x, \varphi, z, t)
$$

is the effective potential for the radial oscillations of the particle. In (3), $\phi=e \Phi(\hat{R}, \varphi, \hat{Z}, \hat{t}) /\left(m \omega_{c}^{2} R_{0}^{2}\right) \quad$ is the normalized electric field potential, whereas $f_{\varphi}$ $\equiv f_{\varphi}(x, z, \varphi, t)=R A_{\varphi}(R, \varphi, Z, t) /\left(R_{0}^{2} B_{0}\right)$ and $f_{z} \equiv f_{z}(x, z, \varphi, t)$ $=A_{z}(R, \varphi, Z, t) /\left(R_{0} B_{0}\right)$ are the normalized components of the vector potential $\mathbf{A}$, respectively.

The Hamiltonian system (1)-(3) for the fast-varying canonical variables $\left(x, \varphi, z, p_{x}, p_{\varphi}, p_{z}\right)$ describes the gyromotion of a particle. The direct integration requires very long computational times since the time steps should be much smaller than the period of the gyro-oscillations. The canonical change from the fast oscillating variables $\left(x, \varphi, z, p_{x}, p_{\varphi}, p_{z}\right)$ to the slowly varying guiding-center ones $\left(\vartheta_{x}, Z, \Phi, I_{x}, P_{z}, P_{\varphi}\right)$ is given by the generating function $F$ $=F\left(x, \varphi, z, I_{x}, P_{\varphi}, P_{z}\right)=z P_{z}+\varphi P_{\varphi}+S$ of mixed variables: old coordinates $(x, \varphi, z)$ and new momenta $\left(P_{x}, P_{\varphi}, P_{z}\right)$. The $x$ component of the new momenta, $I_{x}$, is an action variable, and the conjugated coordinate $\vartheta_{x}$ is an angle variable:

$$
I_{x}=\frac{1}{2 \pi} \oint_{C} p_{x} d x, \quad \vartheta_{x}=\frac{\partial S}{\partial I_{x}} .
$$

The integral is taken along the closed curve $C$ of constant $\mathcal{H}=\mathcal{H}\left(x, z, \varphi, p_{x}, p_{z}, p_{\varphi}, t\right)$ on the $\left(x, p_{x}\right)$ plane. The generating function $S \equiv S\left(x, I_{x}, \epsilon z, \epsilon P_{z}, \epsilon \varphi, \epsilon P_{\varphi}, \epsilon t\right)$ is

$$
S=\int^{x} p_{x}\left(x^{\prime} ; I_{x}, z, P_{z}, \varphi, P_{\varphi}, t\right) d x^{\prime},
$$

where $p_{x}=\sqrt{2}\left[\mathcal{H}-U\left(x ; z, \varphi, P_{z}, P_{\varphi}, t\right)\right]^{1 / 2} ; \epsilon$ is used to designate the smallness of order $\rho_{x} / L$, where $\rho_{x}$ is the amplitude of radial gyro-oscillations and $L$ is a spatial scale of the system.

For the small radial gyroexcursions, $\rho_{x} \ll L$, the effective potential $U$ can be approximated by the Taylor series expansion near its minimal value $x=x_{c}\left(U^{\prime}\left(x_{c}\right)=0\right)$ :

$$
U\left(x ; z, \varphi, P_{z}, P_{\varphi}, t\right)=U_{0}+\frac{1}{2} \omega_{x}^{2}\left(x-x_{c}\right)^{2}+\sum_{k \geqslant 2} a_{k}\left(x-x_{c}\right)^{k},
$$

where $U_{0}=U\left(x=x_{c}, z, \varphi, P_{z}, P_{\varphi}, t\right), x_{c}=x_{c}\left(z, \varphi, P_{z}, P_{\varphi}, t\right)$, and $\omega_{0}=\omega_{x}\left(x_{c}, z, \varphi, P_{z}, P_{\varphi}, t\right)$ are slowly varying functions of the their arguments $z, \varphi, P_{z}, P_{\varphi}, t$. The quantity $\omega_{x}=\sqrt{U_{0}^{\prime \prime}}$ is the frequency of small-amplitude radial oscillations,

$$
\omega_{x}=\left.\left(\frac{\partial^{2} U}{\partial x^{2}}\right)^{1 / 2}\right|_{x=x_{c}} ^{1 / 2}
$$

The equation for the minimum $x_{c}$, coinciding with the radial coordinate of a guiding-center, may be written as

$$
u_{\varphi}^{2}+u_{\varphi} \frac{\partial f_{\varphi}}{\partial x}+(1+x) u_{z} \frac{\partial f_{z}}{\partial x}-(1+x) \frac{\partial \phi}{\partial x}=0,
$$

where $u_{\varphi}=\left(p_{\varphi}-f_{\varphi}\right) /(1+x)$ and $u_{z}=p_{z}-f_{z}$ are the normalized velocities. For a typical tokamak plasma the normalized frequency $\omega_{x} \sim 1$ and the radial gyro-oscillation frequency $\omega_{R}=\omega_{x} \omega_{c}$ are of the same order as the reference gyrofrequency $\omega_{c}$.
The new Hamiltonian $H=\mathcal{H}+\partial S / \partial t$ for the slow-varying guiding-center variables $\left(\vartheta_{x}, Z, \Phi, I_{x}, P_{z}, P_{\varphi}\right)$ may be presented as a sum

$$
H=H_{0}+\epsilon H_{1} \text {. }
$$

The first term $H_{0}$ of the Hamiltonian does not depend on the fast gyrophase $\vartheta_{x}$ :

$$
H_{0}=\omega_{x}\left(Z, \Phi, P_{z}, P_{\varphi}, t\right) I_{x}+U_{0}\left(Z, \Phi, P_{z}, P_{\varphi}, t\right) .
$$

The small second term $\epsilon H_{1}$ depends on the phase $\vartheta_{x}$ of the radial gyro-oscillations. It was shown in Ref. 31 that for typical tokamak plasma parameters the action variable $I_{x}$ is a good adiabatic invariant of motion, similar to the magnetic moment $\mu=m v_{\perp}^{2} / 2 B$. Moreover, the gyrofrequency $\omega_{R}$ is much larger than both, the toroidal and poloidal transit frequencies of drift motion. Under these conditions one can neglect the small term $\epsilon H_{1}$ containing the gyrophase $\vartheta_{x}$, and the Hamiltonian equations are reduced to the 4D system of equations for the guiding-center variables $Z, \Phi, P_{Z}, P_{\varphi}$ :

$$
\begin{array}{ll}
\frac{d Z}{d t}=\frac{\partial H_{0}}{\partial P_{z}}, & \frac{d P_{z}}{d t}=-\frac{\partial H_{0}}{\partial Z}, \\
\frac{d \Phi}{d t}=\frac{\partial H_{0}}{\partial P_{\varphi}}, & \frac{d P_{\varphi}}{d t}=-\frac{\partial H_{0}}{\partial \Phi} .
\end{array}
$$

The action variable $I_{x}$ is considered as a constant of motion.

The radial center $x_{c}$ and the frequency $\omega_{x}$ are slowly varying functions of the new canonical variables $Z, \Phi, P_{Z}, P_{\varphi}$, and time $t$, i.e., $x_{c}=x_{c}\left(Z, \Phi, P_{Z}, P_{\varphi}, t\right)$ and $\omega_{x}$ $=\omega_{x}\left(Z, \Phi, P_{Z}, P_{\varphi}, t\right)$. The relations between the coordinates $x, z, \varphi$ of the particle and the guiding-center coordinates $x_{c}, Z, \Phi$ are

$$
\begin{aligned}
& x=x_{c}+\rho_{x} \sin \vartheta_{x}, \\
& z=Z-\frac{\partial S}{\partial P_{z}}=Z+\rho_{x} \omega_{0} \frac{\partial x_{c}}{\partial P_{z}} \cos \vartheta_{x}, \\
& \varphi=\Phi-\frac{\partial S}{\partial P_{\varphi}}=\Phi+\rho_{x} \omega_{0} \frac{\partial x_{c}}{\partial P_{\varphi}} \cos \vartheta_{x} .
\end{aligned}
$$

\section{A. Simplified form of the guiding-center equations}

One can significantly simplify the equations of motion (9)-(11) for the guiding-center using the fact that the poloidal magnetic field $B_{Z}$ and the particle velocity $\mathbf{v}$ are much smaller than the toroidal field $B_{0}$ and the reference velocity $\omega_{c} R_{0}$, respectively. Neglecting small deviations of the toroidal field $B_{\varphi}$ with respect to $B_{0} R_{0} / \hat{R}$, one can set $A_{z}(\hat{R})$ $=-B_{0} R_{0} \ln \left(\hat{R} / R_{0}\right)$. Omitting small terms proportional to $u^{2}$ $\ll 1,\left|b_{z} u\right| \ll 1, b_{z}^{2} \ll 1 \quad\left(u=|\mathbf{v}| / \omega_{c} R_{0}, b_{z}=B_{Z} / B_{0}\right)$ in Eqs. (7) and (6), the equation for the radial guiding center $x_{c}$ is reduced to 


$$
p_{z}+\ln (1+x)-(1+x) E_{x}=0,
$$

and the gyrofrequency $\omega_{x}$ is determined only by the toroidal magnetic field $B_{\varphi}=1 /(1+x)$ and the electric field $E_{x}$ $=-\partial \phi / \partial x$ :

$$
\omega_{0} \approx \frac{1}{1+x_{c}}\left[1-\left(1+x_{c}\right) E_{x}-\left(1+x_{c}\right)^{2} \frac{\partial E_{x}}{\partial x}\right]^{1 / 2} .
$$

The Hamiltonian (9) also takes a simpler form,

$$
\begin{aligned}
H_{0}\left(I_{x}, Z, P_{z}, \Phi, P_{\varphi}\right)= & \omega_{0}\left(Z, \Phi, P_{z}, t\right) I_{x}+\frac{\left(P_{\varphi}-f_{\varphi}\right)^{2}}{2\left(1+x_{c}\right)^{2}} \\
& +\phi\left(x_{c}, Z, \Phi, t\right),
\end{aligned}
$$

where $f_{\varphi}=f_{\varphi}\left(x_{c}, Z, \Phi, t\right), x_{c}=x_{c}\left(Z, \Phi, P_{z}, t\right)$. The corresponding Hamiltonian equations read

$$
\begin{aligned}
& \frac{d Z}{d t}=\frac{\partial \omega_{0}}{\partial P_{z}} I_{x}+\frac{\partial U}{\partial x_{c}} \frac{\partial x_{c}}{\partial P_{z}}, \\
& \frac{d P_{z}}{d t}=-\frac{\partial \omega_{0}}{\partial Z} I_{x}+\frac{\left(P_{\varphi}-f_{\varphi}\right)}{\left(1+x_{c}\right)^{2}} \frac{\partial f_{\varphi}}{\partial Z}-\frac{\partial \phi}{\partial Z}-\frac{\partial U}{\partial x_{c}} \frac{\partial x_{c}}{\partial Z}, \\
& \frac{d \Phi}{d t}=\frac{\left(P_{\varphi}-f_{\varphi}\right)}{\left(1+x_{c}\right)^{2}}, \\
& \frac{d P_{\varphi}}{d t}=-\frac{\partial \omega_{0}}{\partial \Phi} I_{x}+\frac{\left(P_{\varphi}-f_{\varphi}\right)}{\left(1+x_{c}\right)^{2}} \frac{\partial f_{\varphi}}{\partial \Phi}-\frac{\partial \phi}{\partial \Phi}-\frac{\partial U}{\partial x_{c}} \frac{\partial x_{c}}{\partial \Phi},
\end{aligned}
$$

where

$$
\frac{\partial U}{\partial x_{c}}=-\frac{\left(P_{\varphi}-f_{\varphi}\right)^{2}}{\left(1+x_{c}\right)^{3}}-\frac{\left(P_{\varphi}-f_{\varphi}\right)}{\left(1+x_{c}\right)^{2}} \frac{\partial f_{\varphi}}{\partial x_{c}}+\frac{\partial \phi}{\partial x_{c}} .
$$

In the absence of the electric field $\left(E_{x} \equiv 0\right)$ we have simple solutions of Eqs. (15) and (16) in the form

$$
\omega_{x}=\frac{1}{1+x_{c}}=e^{P_{z}}, \quad x_{c}=e^{-P_{z}}-1 .
$$

The relations between the particle coordinates and the guiding-center coordinates also take more simple forms: $x$ $=x_{c}+\rho_{x} \sin \vartheta_{x}, z=Z+\rho_{x} \cos \vartheta_{x}, \varphi=\Phi$. The system of equations (18)-(21) is then further simplified because the frequency $\omega_{x}$ and the guiding-center $x_{c}$ depend only on $P_{z}$.

\section{B. Hamiltonian formalism for passing particles}

For passing particles the derivative $\dot{\varphi} \equiv d \varphi / d t$ does not change its sign during the motion, i.e., $\dot{\varphi}>0$ (or $\dot{\varphi}<0$ ). There exists a small portion of chaotic, barely passing particles. These particles may chaotically transfer from the passing regime to the trapped one, and vice versa. For the majority of passing particles, however, the guiding-center equations can be formulated by choosing the toroidal angle $\varphi$ as an independent time-like variable. The corresponding Hamiltonian function $K$ will be $-P_{\varphi}$. The original time variable $t$ becomes a dependent coordinate. Its conjugate momentum is equal to $h=-H$. The Hamiltonian equations can now be formulated in the four-dimensional phase space $Z, t, P_{z}, h$,

$$
\begin{aligned}
& \frac{d Z}{d \varphi}=\frac{\partial K}{\partial P_{z}}, \quad \frac{d P_{z}}{d \varphi}=-\frac{\partial K}{\partial Z}, \\
& \frac{d t}{d \varphi}=\frac{\partial K}{\partial h}, \quad \frac{d h}{d \varphi}=-\frac{\partial K}{\partial t},
\end{aligned}
$$

with the Hamiltonian function

$$
K \equiv-P_{\varphi}=F-\sigma\left(1+x_{c}\right) \sqrt{2\left(-h-\omega_{x} I_{x}-\phi\right)},
$$

where $F\left(Z, P_{z}, t, h, \varphi\right)=-f_{\varphi}\left(x_{c}, Z, \varphi, t\right)$, and $\sigma=v_{\varphi} /\left|v_{\varphi}\right|$ stands for the direction of particle movement along the toroidal axis. We have $\sigma=+1$ for co-passing particles and $\sigma=-1$ for counterpassing particles. The guiding-center coordinate $x_{c}$ and the frequency $\omega_{x}$ are determined by Eqs. (15) and (16). Because of the implicit dependence of the Hamiltonian $K$ on the variables $Z, t, P_{z}$ through $x_{c}$, the derivatives on the righthand side of Eqs. (24) should be calculated accordingly.

In the absence of an electric field $(\phi \equiv 0)$, the Hamiltonian equations can be further simplified. Then, the coordinate $x_{c}$ and the frequency $\omega_{x}$ are determined only by the momentum $P_{z}$ [see Eq. (23)], and the equations of motion take the form

$$
\begin{aligned}
& \frac{d Z}{d \varphi}=-e^{-P_{z}}\left[\frac{\partial f_{\varphi}}{\partial x}-\sigma\left(2 h+e^{\left.P_{z} I_{x}\right)}\right] \sqrt{2\left(-h-e^{\left.P_{z} I_{x}\right)},\right.}\right. \\
& \frac{d P_{z}}{d \varphi}=-\frac{\partial f_{\varphi}}{\partial Z},
\end{aligned}
$$

$$
\frac{d t}{d \varphi}=\frac{\sigma e^{-P_{z}}}{\sqrt{2\left(-h-e^{\left.P_{z} I_{x}\right)}\right.}}, \quad \frac{d h}{d \varphi}=-\frac{\partial f_{\varphi}}{\partial t} .
$$

One should note that for the co-passing particles $(\sigma=+1)$ the Hamiltonian equations should be integrated along a positive direction of the toroidal angle $\varphi$, and vice versa for counterpassing particles. From the first set of Eqs. (27) one can recover the magnetic field line equations by setting $\sigma=0$.

\section{MAPPING OF DRIFT SURFACES}

\section{A. Equilibrium magnetic field}

Consider a model of the equilibrium tokamak plasma with nested, circular magnetic surfaces which takes into account the outward (Shafranov) shift of magnetic surfaces due to effects of the plasma pressure and electric current (see Appendix A and Ref. 35 for details). The shift $\Delta(\rho)=R_{p}(\rho)$ $-R_{p}(a)$ of the center $R_{p}(\rho)$ of the magnetic surface of radius $\rho$ from the center of the last magnetic surface $R_{p}(a)$ with a radius $a$ is mainly determined by the plasma $\beta_{\text {pol }}$, i.e., the ratio of the plasma pressure to the pressure of the poloidal field $B_{\theta}$ [see Eq. (A5)]. The safety factor $q(\rho)$ is assumed to have a parabolic radial profile given by

$$
q(\rho)=q_{0}+\left(q_{a}-q_{0}\right) \frac{\rho^{2}}{a^{2}},
$$

where $q_{0}$ and $q_{a}$ are values of the safety factor at the magnetic axis and at the plasma radius, respectively. 
Furthermore, for the numerical evaluations we choose the following parameters: toroidal magnetic field $B_{0}$ $=2.25 \mathrm{~T}$, major radius of the torus $R_{0}=175 \mathrm{~cm}$, minor radius $a=46.7 \mathrm{~cm}$, geometrical center of the plasma $R_{a}=174 \mathrm{~cm}$, $q_{0}=0.75, q_{a}=3.5$.

\section{B. Effect of magnetic perturbations}

We study the effect of nonaxisymmetric magnetic perturbations on the guiding center motion of particles. Specifically, we consider the magnetic perturbation in the DED of the TEXTOR, which is intended to create a stochastic magnetic field at the plasma edge. The corresponding model of the perturbation field is given in Appendix A. A detailed description of this model and the structure of the magnetic field is given in Refs. 35, 45, and 46. We will restrict ourselves to the single particle dynamics, ignoring Coulomb collisions and turbulent electrostatic fluctuations. This will allow us to study the influence of the stochastic magnetic field on particle transport without shadowing it with the effects of collisions and turbulence.

According to (A1) and (A6) the normalized $\varphi$ component of the vector potential $f_{\varphi}$ can be presented as

$$
f_{\varphi}(x, z, \varphi, t)=f_{0}(x, z)+\epsilon f_{1}(x, z, \varphi, t),
$$

where

$$
\begin{aligned}
& f_{0}(x, z)=\int \frac{d \psi}{q(\rho(\psi))}, \\
& \psi=\frac{\rho^{2}}{2 R_{0}^{2}}=\frac{1}{2}\left[\left(1+x-R_{a} / R_{0}-\Delta(\rho) / R_{0}\right)^{2}+z^{2}\right], \\
& \epsilon f_{1}(x, z, \varphi, t)=\epsilon \sum_{m} f_{m}(r, \theta) \cos (m \theta-n \varphi+\Omega t), \\
& f_{m}(r, \theta)=-\bar{r}_{c} g_{m} m^{-1} \sqrt{1+\bar{r} \cos \theta}\left(\frac{\bar{r}}{\bar{r}_{c}}\right)^{m} .
\end{aligned}
$$

Here $\epsilon=B_{c} / B_{0}$ stands for the perturbation parameter, $\bar{r}=\left(x^{2}\right.$ $\left.+z^{2}\right)^{1 / 2}$ is the normalized minor radius, $\left(\bar{r}_{c}=r_{c} / R_{0}\right), \theta$ is a poloidal angle. Suppose that the equilibrium electric field potential $\phi$ is toroidally axisymmetric, i.e., it does not depend on $\varphi$ and $\phi=\phi(x, z)$. Using (30), the Hamiltonian (17) for the guiding-center motion may be rewritten in the form $H=H_{0}\left(Z, P_{z}, P_{\varphi}\right)+\epsilon H_{1}\left(Z, \Phi, P_{z}, P_{\varphi}, t\right)$, where

$$
H_{0}=\omega_{0}\left(Z, P_{z}\right) I_{x}+\frac{\left[P_{\varphi}-f_{0}\left(x_{c}, Z\right)\right]^{2}}{2\left(1+x_{c}\right)^{2}}+\phi\left(x_{c}, Z\right)
$$

describes the guiding-center motion in the unperturbed equilibrium field, and

$$
\epsilon H_{1}=-\epsilon \frac{\left[P_{\varphi}-f_{0}\left(x_{c}, Z\right)\right] f_{1}\left(x_{c}, Z, \Phi, t\right)}{\left(1+x_{c}\right)^{2}}
$$

corresponds to the perturbation due to magnetic perturbations in the first order of $\epsilon$.

The effect of magnetic field perturbations on passing particles can be also analyzed using the Hamiltonian equa- tions (24) with a toroidal angle $\varphi$ as an independent variable. Using (30), the Hamiltonian (25) can be written as

$$
K=K_{0}\left(Z, P_{z}, h\right)+\epsilon K_{1}\left(Z, t ; P_{z}, h ; \varphi\right),
$$

where

$$
K_{0}=-f_{0}\left(x_{c}, Z\right)-\sigma\left(1+x_{c}\right) \sqrt{2\left(-h-\omega_{x} I_{x}-\phi\right)}
$$

describes the drift motion in the equilibrium magnetic field, and

$$
\epsilon K_{1}\left(Z, t ; P_{z}, h, \varphi\right)=-f_{1}\left(x_{c}\left(P_{z}\right), Z, \varphi, t\right),
$$

corresponds to magnetic perturbations.

\section{Unperturbed drift surfaces}

In the absence of nonaxisymmetric magnetic and electric perturbations there are two independent integrals of motion: the particle energy $E=H_{0}$ and the momentum $P_{\varphi}$. Then the system is equivalent to an integrable one-degree-of-freedom system with Hamiltonian $\tilde{H}\left(Z, P_{z}\right)=H_{0}\left(Z, P_{z} ; P_{\varphi}\right)$. The guiding-center orbits lie on toroidal drift surfaces which do not coincide with the magnetic surfaces.

According to Eq. (38) the drift surfaces are determined by the surfaces of constant energy, i.e., $f_{D}(x, Z)=$ const, where $f_{D}(x, Z) \equiv K_{0}\left(Z, P_{z}(x) ; h\right)$. On the other hand, the magnetic surfaces are determined by $f_{M}(x, Z)=-f_{0}(x, Z)=$ const, i.e., by the first term on the right-hand side of Eq. (38). Therefore, the deviation of the drift surfaces from the magnetic ones depends on several factors, particularly on the energy $h$ of particles and the direction $\sigma$ of the particle velocity. The estimation of this deviation has been given in Ref. 31.

The dependence of drift surfaces on $\sigma$ has a simple explanation. It is known that the drift velocity $\mathbf{u}_{d}$ due to $\nabla \mathbf{B}$ and the curvature drifts does not depend on the directions of particle velocity, i.e., $\mathbf{u}_{d} \propto v_{\|}^{2}+v_{\perp}^{2} / 2$ (see, e.g., Ref. 48, p. 47). The total velocity of guiding centers, $\mathbf{v}_{c}$, is then given by the sum of $\mathbf{u}_{d}$ and the parallel velocity $\mathbf{v}_{\|}$, i.e., $\mathbf{v}_{c}=\mathbf{v}_{\|}+\mathbf{u}_{d}$. When the direction of particle motion is changed, $\sigma \leftrightarrow-\sigma$, the velocity $\mathbf{v}_{c}^{(\sigma)}$ is changed to $\mathbf{v}_{c}^{(-\sigma)}=-\mathbf{v}_{\|}+\mathbf{u}_{d}$, i.e., $\mathbf{v}_{c}^{(\sigma)} \neq-\mathbf{v}_{c}^{(-\sigma)}$. Therefore, the guiding center orbits of particles moving in opposite directions (at a given energy) do not coincide.

In Fig. 1 the deviations of the guiding-center orbits from the magnetic surfaces are shown for the co-passing (curves 1 ) and the counterpassing particles (curves 2) on the poloidal section $(r, \theta)$, curves 3 correspond to the magnetic surfaces. The total particle energy is $E=1 \mathrm{keV}$, the relative energy of radial oscillations $\lambda=I_{x} / E=0.5$, and the plasma parameter $\beta_{\mathrm{pol}}=0.5$.

\section{Equations of motion in action-angle variables}

It is useful to formulate the equations of motion in action-angle variables to study the regular and chaotic dynamics of system. In Appendix B we introduced these variables for the Hamiltonian system (17). Here we formulate the Hamiltonian system (24) for the passing particles in the corresponding variables.

In the absence of nonaxisymmetric magnetic and electric perturbations there are two independent integrals of motion: 


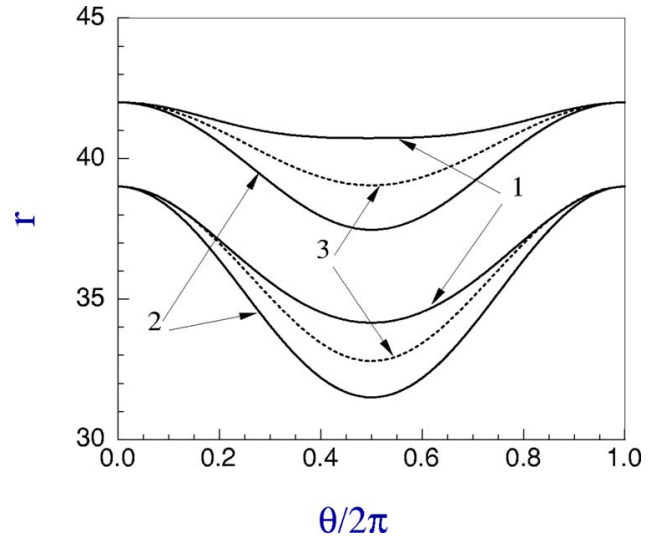

FIG. 1. (Color online) Deviations of the drift surfaces from the magnetic surfaces, for co-passing (curves 1) and counterpassing (curves 2) particles, on the poloidal section $(r, \theta)$. Curves 3 denote the magnetic surfaces. The two sets of curves correspond to different initial conditions.

$C_{z}=$ const related to the drift motion in the poloidal plane, and $h=-H=$ const. For the motion on the poloidal $(x, z)$ plane one can use the action variables $I_{z}$ as defined in (B1). We introduce canonical angle variables $\vartheta_{z}, T$ conjugated to the action variables $I_{z}, h$ :

$$
\begin{aligned}
& \vartheta_{z}=\frac{\partial S}{\partial I_{z}}=\frac{\partial}{\partial I_{z}} \int^{Z} P_{z}\left(Z^{\prime}, I_{z}, h\right) d Z^{\prime}, \\
& T=\frac{\partial S}{\partial h}=t+\frac{\partial}{\partial h} \int^{Z} P_{z}\left(Z^{\prime}, I_{z}, h\right) d Z^{\prime},
\end{aligned}
$$

where $S=h t+\int{ }^{Z} P_{z}\left(Z^{\prime}, I_{z}, h\right) d Z^{\prime}$ is the corresponding generating function. In the new variables the unperturbed Hamiltonian $K_{0}$ depends only on the action $I_{z}$ and the energy variable $h, K_{0}=K_{0}\left(I_{z}, h\right)$. The angle variable $\vartheta_{z}$ and the new time variable $T$ are linear functions of the toroidal angle $\varphi, \vartheta_{z}$ $=\nu_{z}\left(I_{z}, h\right) \varphi+\vartheta_{z 0}, T=\nu_{t}\left(I_{z}, h\right) \varphi+T_{0}$. Here $\nu_{z}$ and $\nu_{t}$ are the corresponding poloidal and "temporal transit frequencies" of drift motion, respectively:

$$
\nu_{z}\left(I_{z}, h\right)=\frac{\partial K_{0}\left(I_{z}, h\right)}{\partial I_{z}}, \quad \nu_{t}\left(I_{z}, h\right)=\frac{\partial K_{0}\left(I_{z}, h\right)}{\partial h} .
$$

The frequency $\nu_{z}$ is the analog of the rotational transform $\iota$ (or the inverse safety factor $q$ ) of magnetic field lines. It has the meaning of the number of poloidal drift rotations per one toroidal turn. The quantity $\nu_{z}^{-1}=q_{\text {eff }}$ will be called the effective safety factor. The temporal transit frequency $\nu_{t}$ has the meaning of the averaged time spent by a particle for the toroidal motion during $\Delta \varphi=1$. Its inverse $\nu_{t}^{-1}$ coincides with the toroidal transit frequency $\omega_{\varphi}$ of drift motion introduced in Sec. III, i.e., $\nu_{t}^{-1}=\omega_{\varphi}\left(I_{z}, I_{\varphi}\right)$. The old variables $Z, P_{z}, t, h$ are periodic functions of both, the angle $\vartheta_{z}$ and the time variable $T$, and can be presented as a Fourier series:

$$
\begin{aligned}
& Z=Z\left(\vartheta_{z}, I_{z}, h\right)=\sum_{m} Z_{m}\left(I_{z}, h\right) \exp \left(i m \vartheta_{z}\right), \\
& P_{z}=P_{z}\left(\vartheta_{z}, I_{z}, h\right)=\sum_{m} P_{m}\left(I_{z}, h\right) \exp \left(i m \vartheta_{z}\right),
\end{aligned}
$$

$$
t=T+\sum_{m} t_{m}\left(I_{z}, h\right) \exp \left(i m \vartheta_{z}\right)
$$

In the new variables the total Hamiltonian (37) can be written as

$$
K=K_{0}\left(I_{z}, h\right)+\epsilon K_{1}\left(\vartheta_{z}, I_{z}, T, h, \varphi\right),
$$

where

$$
K_{1}=\sum_{m} K_{m n}\left(I_{z}, h\right) \cos \left(m \vartheta_{z}+\Omega T-n \varphi\right)
$$

is the perturbed Hamiltonian with the Fourier coefficients

$$
\begin{aligned}
K_{m n}\left(I_{z}, h\right)= & \frac{1}{\pi} \int_{0}^{2 \pi} \sum_{m} f_{m}\left(r\left(\vartheta_{z}, I_{z}, h\right), \theta\left(\vartheta_{z}, I_{z}, h\right)\right) \\
& \times \cos m \theta\left(\vartheta_{z}, I_{z}, h\right) \cos \left(m \vartheta_{z}\right) d \vartheta_{z} .
\end{aligned}
$$

In the presence of nonaxisymmetric perturbations (39) the equations of drift motion (in variables $\vartheta, T, I_{z}, h$ ) are

$$
\begin{aligned}
& \frac{d \vartheta_{z}}{d \varphi}=\nu_{z}\left(I_{z}, h\right)+\epsilon \frac{\partial K_{1}}{\partial I_{z}}, \quad \frac{d I_{z}}{d \varphi}=-\epsilon \frac{\partial K_{1}}{\partial \vartheta_{z}}, \\
& \frac{d T}{d \varphi}=\nu_{t}\left(I_{z}, h\right)+\epsilon \frac{\partial K_{1}}{\partial h}, \quad \frac{d h}{d \varphi}=-\epsilon \frac{\partial K_{1}}{\partial T} .
\end{aligned}
$$

\section{E. Symplectic mapping}

We will integrate Eqs. (49) and (50) using the new symplectic mapping method proposed in Refs. 16, 17, and 19. From the computational point of view, mappings run much faster than conventional integration methods. Maps with large "integration steps" can achieve the same accuracy of computations as the conventional methods with much smaller integration steps.

The symplectic mapping for the Hamiltonian system (46)-(50) is constructed in the following way. We introduce poloidal sections $\varphi_{k}=k(2 \pi / s)(k=0, \pm 1, \pm 2, \ldots)$, where $s$ $\geqslant 1$ is an integer number. Let $\vartheta_{k}, I_{k}, t_{k}, h_{k}$ be the intersection points of the drift orbit $I_{z}(\varphi), h(\varphi), \vartheta_{z}(\varphi), T(\varphi)$ with the poloidal section $\varphi=\varphi_{k}$. The forward 4D map

$$
\left(I_{k+1}, \vartheta_{k+1}, T_{k+1}, h_{k+1}\right)=\hat{T}_{+}\left(I_{k}, \vartheta_{k}, T_{k}, h_{k}\right),
$$

connecting the intersection points of the orbit at $\varphi=\varphi_{k}$ with the ones at $\varphi=\varphi_{k+1}$, describes the dynamics of co-passing particles. On the other hand, the backward 4D map

$$
\left(I_{k-1}, \vartheta_{k-1}, T_{k-1}, h_{k-1}\right)=\hat{T}_{-}\left(I_{k}, \vartheta_{k}, T_{k}, h_{k}\right)
$$

describes the dynamics of counterpassing particles. The symplectic map (51), based on the Hamiltonian (46), has the following explicit form given in Ref. 17:

$$
\begin{aligned}
& J_{k}=I_{k}-\epsilon \frac{\partial S_{k}}{\partial \vartheta_{k}}, \quad \Theta_{k}=\vartheta_{k}+\epsilon \frac{\partial S_{k}}{\partial J_{k}}, \\
& \mathcal{H}_{k}=h_{k}-\epsilon \frac{\partial S_{k}}{\partial T_{k}}, \quad \mathcal{T}_{k}=T_{k}+\epsilon \frac{\partial S_{k}}{\partial \mathcal{H}_{k}},
\end{aligned}
$$




$$
\begin{aligned}
& J_{k+1}=J_{k}, \quad \Theta_{k+1}=\Theta_{k}+\nu_{z}\left(J_{k}, \mathcal{H}_{k}\right) \Delta \varphi, \\
& \mathcal{H}_{k+1}=\mathcal{H}_{k}, \quad \mathcal{T}_{k+1}=\mathcal{T}_{k}+\nu_{t}\left(J_{k}, \mathcal{H}_{k}\right) \Delta \varphi, \\
& I_{k+1}=J_{k+1}+\epsilon \frac{\partial S_{k+1}}{\partial \vartheta_{k+1}}, \quad \vartheta_{k+1}=\Theta_{k}-\epsilon \frac{\partial S_{k+1}}{\partial J_{k+1}}, \\
& h_{k+1}=\mathcal{H}_{k+1}+\epsilon \frac{\partial S_{k+1}}{\partial T_{k+1}}, \quad T_{k+1}=\mathcal{T}_{k}-\epsilon \frac{\partial S_{k+1}}{\partial \mathcal{H}_{k+1}} .
\end{aligned}
$$

The functions $S_{k}$ and $S_{k+1}$ are the values of the generating function $S(\vartheta, J, T, \mathcal{H}, \varphi, \epsilon)$ at $\varphi \rightarrow \varphi_{k}+0$ and $\varphi \rightarrow \varphi_{k+1}-0$, respectively. In Eqs. (53)-(58) the quantity $\Delta \varphi=\varphi_{k+1}-\varphi_{k}$ $=2 \pi / s$ is the map step along the toroidal axis $\varphi$. In the first order of the perturbation parameter $\epsilon$, the generating function $S(\vartheta, J, T, \mathcal{H}, \varphi, \epsilon)$ is determined by the integral

$$
S=-\int_{\varphi_{0}}^{\varphi} K_{1}\left(\vartheta_{z}\left(\varphi^{\prime}\right), J, T\left(\varphi^{\prime}\right), \mathcal{H}, \varphi^{\prime}\right) d \varphi^{\prime}+\mathcal{O}(\mu),
$$

where the running toroidal angle $\varphi$ and the free parameter $\varphi_{0}$ are located in the interval $\left[\varphi_{k}, \varphi_{k+1}\right]$. In (59) the integral is taken along the unperturbed orbit $\vartheta_{z}\left(\varphi^{\prime}\right)=\nu_{z}\left(\varphi^{\prime}-\varphi\right)+\vartheta_{z}(\varphi)$, $t\left(\varphi^{\prime}\right)=\nu_{t}\left(\varphi^{\prime}-\varphi\right)+t(\varphi)$. The term $\mathcal{O}(\mu)$ in (59) stands for the corrections of the order of the small parameter $\mu=\epsilon \mid \varphi$ $-\left.\varphi_{0}\right|^{\delta} \ll 1,(\delta \geqslant 1)$.

Using the Fourier representation (47) of $K_{1}$, the generating function $S$ can be presented in the form

$$
\begin{aligned}
S= & -\left(\varphi-\varphi_{0}\right) \sum_{m} K_{m}(J, \mathcal{H})\left[a\left(\alpha_{m}\right) \sin \left(m \vartheta_{z}-n \varphi+\Omega T\right)\right. \\
& \left.+b\left(\alpha_{m}\right) \cos \left(m \vartheta_{z}-n \varphi+\Omega T\right)\right],
\end{aligned}
$$

where $\alpha_{m}=\left(m \nu_{z}(J, \mathcal{H})-n+\Omega \nu_{t}(J, \mathcal{H})\right)\left(\varphi-\varphi_{0}\right)$ and

$$
a(x)=\frac{1-\cos x}{x}, \quad b(x)=\frac{\sin x}{x} .
$$

The 4D map (52) for the counterpassing particles has a similar form. The accuracy of the mapping depends on the free parameter $\varphi_{0}$. The highest accuracy may be achieved when $\varphi_{0}$ is located exactly in the middle of the interval $\left[\varphi_{k}, \varphi_{k+1}\right]$, i.e., $\varphi_{0}=\left(\varphi_{k}+\varphi_{k+1}\right) / 2$.

For time-independent perturbations $(\Omega=0)$ the particle energy $H$ is an integral of motion. For this case the 4D map (53)-(58) is reduced to a 2D map for the action-angle variables $\vartheta, I:\left(I_{k+1}, \vartheta_{k+1}\right)=\hat{T}_{+}\left(I_{k}, \vartheta_{k}\right)$. This map is determined by the equations on the first lines in (53)-(58). The increment in time $\Delta T=T_{k+1}-T_{k}$ over one step of the map is determined by

$$
\Delta T=\nu_{t}\left(J_{k}, \mathcal{H}_{k}\right) \Delta \varphi+\epsilon\left(\frac{\partial S_{k}}{\partial \mathcal{H}_{k}}-\frac{\partial S_{k+1}}{\partial \mathcal{H}_{k+1}}\right) .
$$

The mapping method requires the knowledge of the transit frequencies $\nu_{z}(J, \mathcal{H}), \nu_{t}(J, \mathcal{H})$ of the unperturbed system, and the Fourier coefficients $K_{m n}(J, \mathcal{H})$ of the perturbed Hamiltonian. Analytical determination of these functions in a toroidal geometry is somehow difficult. In order to avoid these difficulties we have calculated these functions numerically according to Eqs. (48) for the rectangular grid coordinates of $J, H: J_{i}=J_{0}+i \Delta J, H_{j}=H_{0}+j \Delta H \quad\left(i=1, \ldots, N_{J}, j\right.$ $\left.=1, \ldots, N_{H}\right)$. This was carried out by integration of the unperturbed Hamiltonian equations (18)-(21) over one poloidal turn on the $(x, z)$ plane. The functions $\nu_{z}(J, \mathcal{H}), \nu_{t}(J, \mathcal{H})$, $K_{m n}(J, \mathcal{H})$ for the arbitrary values of $J$ are interpolated using the cubic splines.

In order to display the orbits in configurational space $(x, z, \varphi)[$ or $(r, \theta, \varphi)]$, the relation to the action-angle variables $J, \vartheta$ have to be found. Making the ansatz

$$
\begin{aligned}
& r=r_{0}+\sum_{m=1}^{M}\left(r_{m}^{(s)}(J, H) \sin m \vartheta+r_{m}^{(c)}(J, H) \cos m \vartheta\right), \\
& \theta=\vartheta+\sum_{m=1}^{M} \alpha_{m}(J, H) \sin m \vartheta,
\end{aligned}
$$

the Fourier coefficients $r_{m}^{(s)}(J), r_{m}^{(s)}(J)$, and $\alpha_{m}(J)$ can be calculated numerically by integrating the unperturbed Hamiltonian system (38) over one poloidal rotation on the $(x, z)$ plane for the grid coordinates corresponding to $J, H$. The values for arbitrary $J, H$ are interpolated by cubic splines.

Maps run much faster than the standard integration schemes (approximately 30-40 times for our problems). We have shown that they reproduce all features of particle dynamics obtained by the direct numerical integration of Hamiltonian equations (18)-(21) when using a fifth order Runge-Kutta scheme.

\section{ONSET OF CHAOTIC DRIFT MOTION}

As long as particles predominantly follow the magnetic field lines, the chaotic drift motion may appear close to the onset of stochasticity of magnetic field lines. However, due to the toroidal drift the guiding centers deviate from the magnetic field (see Fig. 1), and thereby the zone of chaotic drift motion may be different from the magnetic one.

The well-known mechanism for the onset of chaotic field line motion is the following. The external $(m, n)$ resonant magnetic perturbations destroy the rational magnetic surfaces $\psi_{m n}$ determined by $q\left(\psi_{m n}\right)=m / n$, forming chains of magnetic islands. The radial widths $W_{m n}$ of these islands grow with the perturbation strength $\epsilon$ as $W_{m n} \propto \epsilon^{1 / 2}$. At a certain level of $\epsilon$ the neighboring islands start to overlap leading the global chaotic behavior of field lines.

For the passing particles, according to (47) and (42), the resonant drift surfaces are determined by the condition

$$
m \nu_{z}\left(I_{z}, \mathcal{H}\right)-n+\sigma \Omega \nu_{t}\left(I_{z}, \mathcal{H}\right)=0 .
$$

[They are also determined by Eq. (B13) for both, passing as well as trapped particles.] For static magnetic perturbations $(\Omega=0)$ the condition can be written similar to the resonant condition for the magnetic surfaces: $q_{\text {eff }}(I, H)=m / n$, where $q_{\mathrm{eff}} \equiv \nu_{z}^{-1}\left(I_{z}, H\right)=\omega_{\varphi}\left(I_{z}, I_{\varphi}\right) / \omega_{z}\left(I_{z}, I_{\varphi}\right)$ is the effective $q$ profile of the drift motion. The latter depends not only on the plasma parameters but also on particle energy $H$, the adiabatic invariant $I_{x}$, as well as on the direction of motion $\sigma=v_{\varphi} /\left|v_{\varphi}\right|$. 

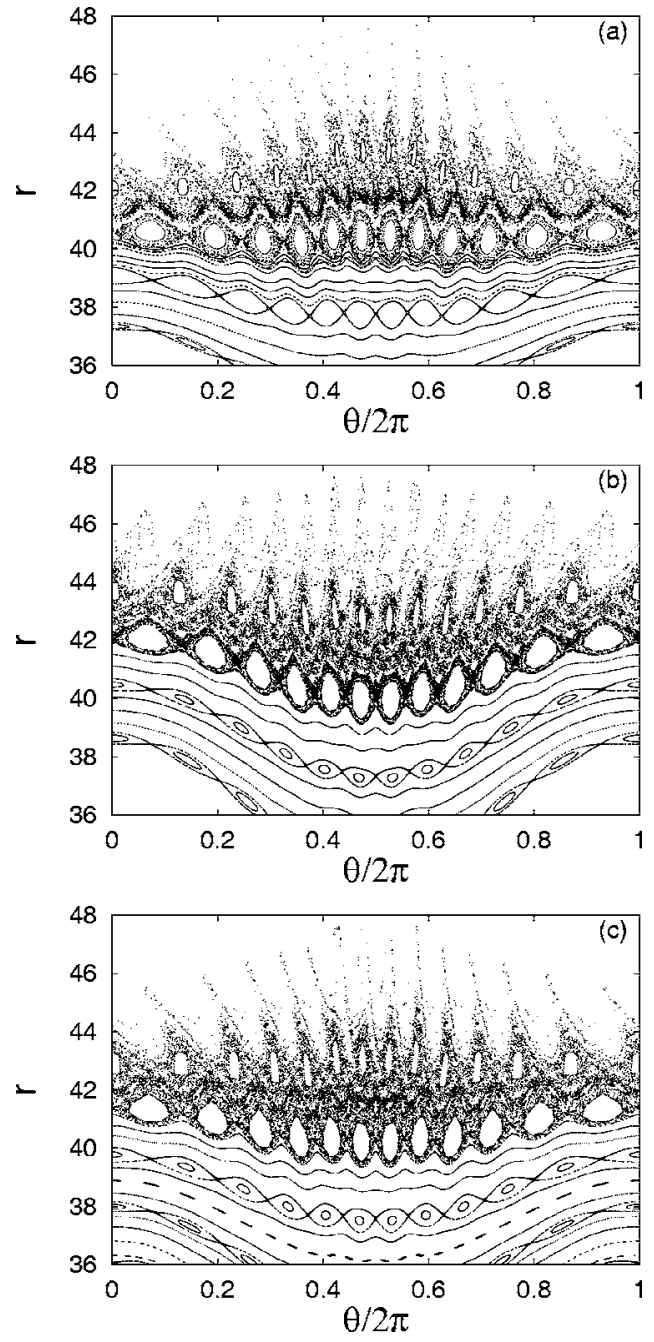

FIG. 2. Poincaré sections of guiding-center motion on the poloidal plane $(r, \theta)$ : (a) co-passing particles; (b) counterpassing particles; (c) magnetic field lines. The parameter values are $E=1 \mathrm{keV}, \lambda=0.4$, and $\beta_{\mathrm{pol}}=0.5$.

\section{A. Poincaré sections}

It is convenient to study the chaotic motion by displaying the crossing points of particle orbits with the poloidal plane $(\theta, r)$ at fixed $\varphi=$ const. Such a set of points, known as the Poincaré section, is shown in Fig. 2 for passing particles with energy $E=1 \mathrm{keV}$, the relative radial energy $\lambda=0.4$, and the plasma $\beta_{\mathrm{pol}}=0.5$.

Figure 2(a) corresponds to co-passing particles, Fig. 2(b) to counterpassing particles, and Fig. 2(c) describes the corresponding Poincaré section of magnetic field lines. Shown are cases of destroyed but nonoverlapped resonant drift surfaces. The radial positions of the resonant drift surfaces on the low field side (LFS) are shifted inwardly for the copassing particles and outwardly for the counterpassing particles, with respect to the corresponding resonant magnetic surfaces. Such a radial shift is increased with the particle energy. Since the perturbation field strongly decays in the inward direction, the widths of drift islands for the counterpassing particles are larger than for the co-passing particles, and they have wider stochastic layers near the separatrix.

The maximum of the poloidal mode spectrum $K_{m n}$ is
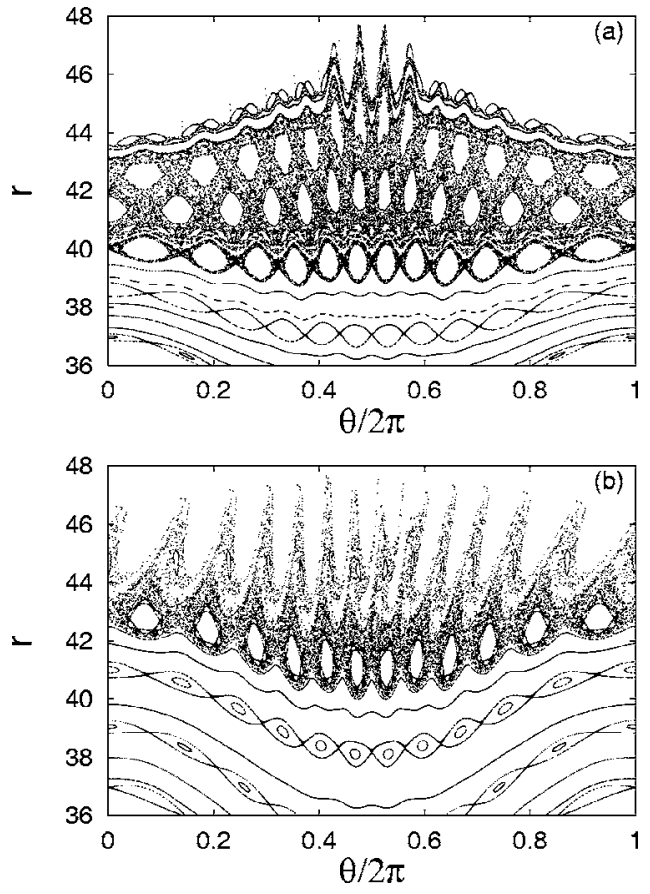

FIG. 3. Poincaré sections of drift orbits in the presence of an electric field: (a) co-passing particles; (b) counterpassing particles. Parameter values are $T_{i}=400 \mathrm{eV}, E=1 \mathrm{keV}, \lambda=0.4$, and $\beta_{\mathrm{pol}}=0.5$.

shifted to the higher $m$ with lowering the plasma $\beta_{\text {pol }}$. It leads to the increase of the ergodization level of particles with the decrease of $\beta_{\mathrm{pol}}$. The highest ergodization level is achieved for $\beta_{\mathrm{pol}}=0$.

\section{B. Influence of a static electric field}

In the presence of an equilibrium radial electric field $E_{r}=-d \Phi(r) / d r$ the particle orbits may significantly deviate from the magnetic field lines due to the combined effect of the toroidal and the $\mathbf{E} \times \mathbf{B}$ drifts. In order to study the effect of the electric field, we have chosen an equilibrium potential with the radial profile $\Phi(r)=-T_{i}\left[1-(r / a)^{4}\right]$.

The $\mathbf{E} \times \mathbf{B}$ drift velocity $\mathbf{v}_{d}$ has a predominant poloidal component $v_{d}^{(\theta)} \approx-c E_{r} / B$, with $E_{r}=4(r / a)^{3} T_{i} / a$. It changes the poloidal transit frequencies of drift motion $\omega_{\theta} \equiv \omega_{z}$ to $\omega_{\theta}^{(+)} \approx \omega_{\theta 0}+v_{d}^{(\theta)} / r=\omega_{\theta 0}-c E_{r} / B r$ for the co-passing particles and $\omega_{\theta}^{(-)} \approx \omega_{\theta 0}+c E_{r} / B r$ for counterpassing particles, respectively, where $\omega_{\theta 0}$ is the poloidal transit frequency in the absence of electric field. Therefore, the effective $q_{\text {eff }}$ profile, defined as $q_{\text {eff }}=\omega_{\varphi} / \omega_{\theta}$, is decreased for the counterpassing particles, and increased for the co-passing particles (with respect to the situation without the radial electric field).

The radial positions of the resonant drift surfaces are further shifted inwardly for the co-passing particles, and outwardly for the counterpassing particles. This further increases the distance between the corresponding $m: n$ resonant drift surfaces of the co-passing and counterpassing particles. It leads to the different ergodization level for the corresponding particles shown in Fig. 3: (a) for the copassing particles, and (b) for the counterpassing particles.

In the first case, the chaotic layer is closed, and the particles are confined. They cannot reach the divertor plates due 

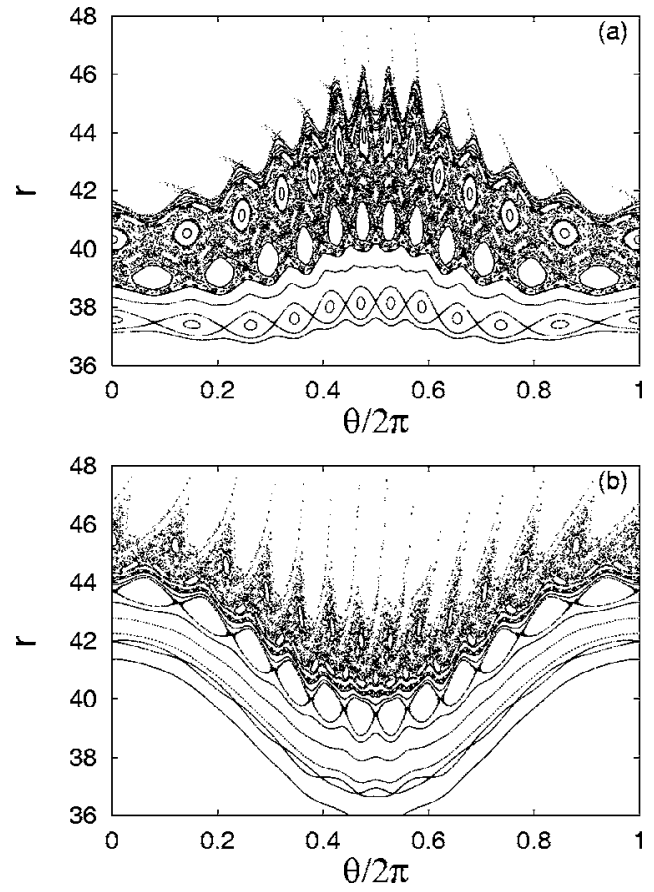

FIG. 4. Poincaré sections of drift orbits of particles with energy $E$ $=10 \mathrm{keV}$ : (a) $\sigma=+1$; (b) $\sigma=-1$. The other parameter values are $\lambda=0.4$ and $\beta_{\mathrm{pol}}=1$.

to a radial transport along the field lines. In the second case, one obtains a well-developed ergodic layer with open orbits hitting the divertor plates.

\section{High-energetic particles}

An increase in the particle energy further modifies the resonant drift surfaces. The resonant drift surfaces of the co-passing particles are further shifted inside the plasma, while for the counterpassing particles they are shifted outside the plasma. The corresponding Poincaré sections are plotted in Fig. 4: (a) for $\sigma=+1$; (b) for $\sigma=-1$.

One can notice that for the high-energetic particles the selective effect of the perturbed magnetic field on the copassing and the counterpassing particles becomes more pronounced. For the co-passing particles the ergodic zone covers a larger area on the LFS of the plasma than for the counterpassing particles. To be more specific, the border of the ergodic zone on the LFS for the first kind of particles is shifted inwardly by $5 \mathrm{~cm}$ in comparison to the second ones. It means that the fast co-passing particles may leave the plasma faster than the counterpassing particles. Such a tendency becomes more pronounced with the further increase of the particle energy. The co-passing particles reach the divertor plates only after a few poloidal turns. On the other hand, the orbits of the counterpassing particles are regular (nonchaotic), and they do not leave the plasma due to the magnetic perturbations. We have to mention that at very high energies, e.g., for runaway particles, a relativistic treatment will become necessary.

\section{Rotating magnetic field}

Next, we consider the effect of rotating magnetic field perturbations. In the presence of a periodically timedependent magnetic field the resonance conditions $q_{\text {eff }}$ $\equiv \omega_{\varphi} / \omega_{\theta}=m / n$ should be replaced by (B13) or (65). Finite rotation frequencies shift the radial positions of the resonant drift surfaces from the static case $(\Omega=0)$. In order to estimate this shift consider the following simple model. Neglecting the effect of the toroidal drift, the effective $q_{\text {eff }}$ may be approximated by the magnetic safety factor $q$, see (29). According to (65), the radial positions of the resonant drift surfaces $\rho_{m n}$ is determined by

$$
\rho_{m n}(\Omega)=\frac{a}{\sqrt{q_{a}-q_{0}}} \sqrt{\frac{m}{n-\sigma \Omega \nu_{t}}-q_{0}} .
$$

Thus, the shift of resonant drift surfaces $\Delta \rho_{m n}=\mid \rho_{m n}(\Omega)$ $-\rho_{m n}(0) \mid$ due to magnetic field rotation depends on its frequency $f=\Omega / 2 \pi$, on the particle energy $E$, and the direction of motion $\sigma$.

We have estimated the shift of drift islands for the TEXTOR-DED parameters with a maximum frequency of rotation $f=10^{4} \mathrm{~Hz}$. For the particle energies, we assume the values $E=50 \mathrm{eV}$ and $E=1 \mathrm{keV}$.

For $E=1 \mathrm{keV}$, the resonant drift surface $(m=12, n=4)$ is approximately shifted inwardly by $2.5 \mathrm{~cm}$ for counterpassing particles, and outwardly to the same amount for co-passing particles. For $E=50 \mathrm{eV}$, the outward shift is $13.7 \mathrm{~cm}$ for $\sigma$ $=+1$, and the inward shift is $8.5 \mathrm{~cm}$ for $\sigma=-1$, respectively.

By changing the frequency $f$ of the rotating magnetic field one can regulate the positions of the resonant drift surfaces as was proposed in Ref. 14. In order to show this we have considered the case $\beta_{\mathrm{pol}}=1$ when the neighboring resonant drift islands do not overlap.

In the case $f \neq 0$ the $O$ - points move on the poloidal plane $(\theta, r)$. The outward shift of $O$-points is $0.11 \mathrm{~cm}$ on the LFS and $0.3 \mathrm{~cm}$ on the HFS, for $f=10^{3} \mathrm{~Hz}, 0.23 \mathrm{~cm}$, and $0.6 \mathrm{~cm}$, respectively, for $f=2 \times 10^{3} \mathrm{~Hz}, 0.45 \mathrm{~cm}$, and $1.3 \mathrm{~cm}$, respectively, for $f=4 \times 10^{3} \mathrm{~Hz}, 1.2 \mathrm{~cm}$, and $3.5 \mathrm{~cm}$, respectively, or $f=10^{4} \mathrm{~Hz}$. Similarly, one can show that for counterpassing particles the resonant drift surfaces are shifted inwardly.

Poincaré sections of drift orbits in the laboratory frame are presented in Fig. 5 for co-passing particles at the rotation frequency $f=10^{4} \mathrm{~Hz}$. The parameters are $\beta_{\mathrm{pol}}=0.5, E$ $=1 \mathrm{keV}$, and $\lambda=0.4$. Particle orbits are integrated up to time $t=10^{-2} \mathrm{~s}$. As can be seen from Fig. 5, the rotation "smears out" the structure of the ergodic zone, making the magnetic islands invisible. It is not easy to reveal the ergodization level from Poincaré sections. However, one can expect that the ergodic zone is shifted outwardly for co-passing particles, while it is shifted inwardly for counterpassing particles.

\section{RADIAL DIFFUSION OF PARTICLES IN THE ERGODIC ZONE}

The chaotic motion of particles at the plasma edge due to the effect the resonant magnetic perturbations acts as an ad- 


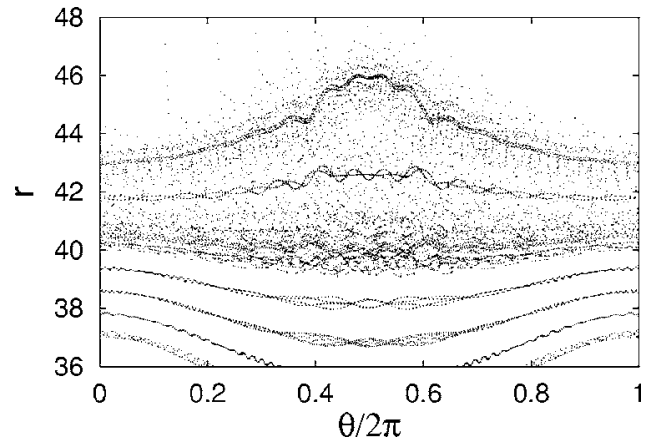

FIG. 5. The same as Fig. 2, but in the presence of time-dependent magnetic perturbations. The parameter values are $E=1 \mathrm{keV}, \beta_{\mathrm{pol}}=0.5, \lambda=0.4, \sigma=1$, $f=10^{4} \mathrm{~Hz}$. Particles are followed up to time $t=0.9 \times 10^{-2} \mathrm{~s}$.

ditional transport to the usual (anomalous) perpendicular energy transport. Below we estimate the radial diffusion rates of particles caused by the chaotic magnetic field lines. Although the description of radial transport in a stochastic layer by diffusion coefficients is not complete, it allows at least to estimate the magnitude of radial transport.

It is known that in a finite ergodic domain one cannot introduce a global radial diffusion coefficient $D_{r}$ by calculating $D_{r}=\sigma_{r}^{2}(t) / 2 t, t \rightarrow \infty$. The latter is defined in an unlimited domain. The radial displacement $\sigma_{r_{0}}^{2}(t)=\left\langle(r-\langle r\rangle)^{2}\right\rangle$ tends to a finite value for $t \rightarrow \infty$ in a finite domain (see, e.g., Ref. 20). The angular bracket $\langle\cdots\rangle$ stands for averaging over a set of initial coordinates uniformly distributed over the drift surface, and $r_{0}$ stands for the radial coordinate of the drift surface on the LFS. One can, however, introduce a local diffusion coefficient $D_{r}(r)$ in the initial linear growth regime of $\sigma_{r}^{2}(t)=2 D(r) t$.

In Fig. 6 the radial profiles of $D(r)$ for ion energy $1 \mathrm{keV}$ and $\lambda=0.4$ are plotted for the static [Fig. 6(a)] and for the rotating [Fig. 6(b)] magnetic field perturbations. In Fig. 6 curves 1 correspond to co-passing particles, curves 2 to counterpassing particles. Curve 3 describes the electron diffusion coefficient $D_{r e}$ for the same energy $1 \mathrm{keV}$, but multiplied by a factor $v_{i} / v_{e}=\left(m_{e} / m_{i}\right)^{1 / 2}=1 / 42.9$. Here $v_{i}$ and $v_{e}$ are ion and electron velocities, respectively. The rotation frequency in case (b) is $f=10^{4} \mathrm{~Hz}$. The corresponding Poincaré sections of ions were shown in Figs. 2 and 5.

We have also calculated the magnetic field diffusion coefficients $D_{\mathrm{FL}}$. The electron diffusion coefficients $D_{r e}$ calculated [curve 3 in Fig. 6(a)] from the drift equations quite well agrees with $D_{r e}=D_{\mathrm{FL}} v_{e}$. The ratio $D_{r e} / D_{\mathrm{FL}}$ is approximately constant and equal to the electron velocity $1.5 \times 10^{7} \mathrm{~m} / \mathrm{s}$. It confirms that electrons predominantly follow magnetic field lines. However, due to toroidal drifts the ion diffusion coefficients $D_{i}$ in the ergodic zone are not simply related to the magnetic diffusivity. Moreover, they depend also on the direction of particle motion. As seen from Fig. 6 in the static case, the ion diffusion coefficients for counterpassing particles are larger than for co-passing particles. One should note that curve 3 [Fig. 6(a)] is proportional to the field line diffusion coefficient $D_{\mathrm{FL}}$, i.e., $D_{r e} v_{i} / v_{e}=D_{\mathrm{FL}} v_{i}=D_{m}$. The diffusion coefficient $D_{m}$ could describe the radial ion diffusion if ions would follow magnetic field lines. However, due to (a)

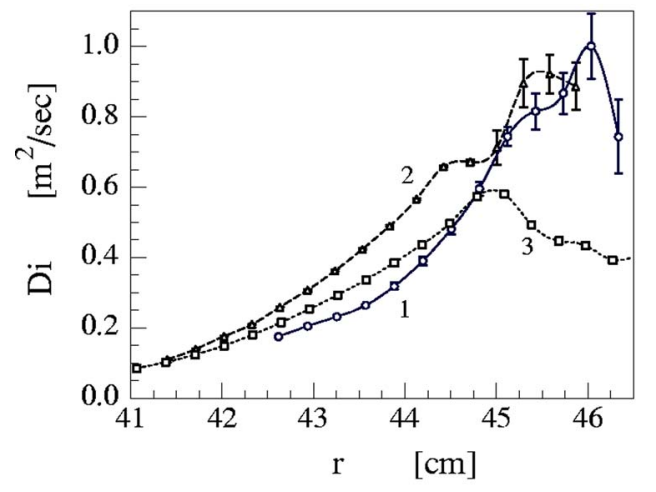

(b)

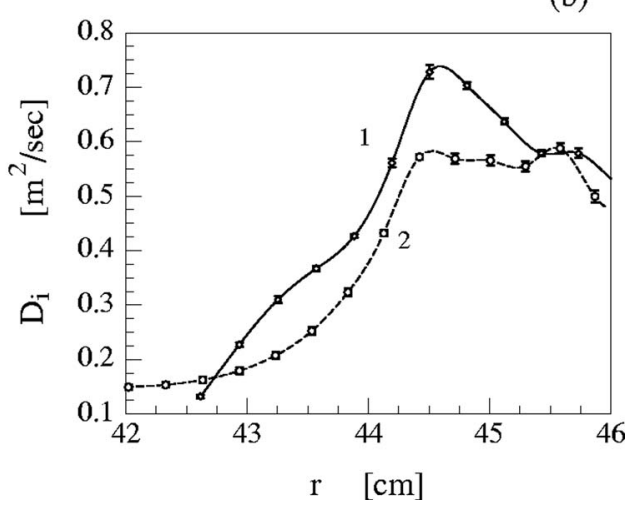

FIG. 6. (Color online) Radial profiles of diffusion coefficients $D_{i}$. (a) Curve 1 corresponds to co-passing particles; curve 2 to counterpassing particles. Curve 3 describes the diffusion coefficient of electrons $D_{e}$ multiplied by the factor $\sqrt{m_{e} / m_{i}} \approx 1 / 42.9$. Parameters are the same as in Fig. 2. (b) The same as (a) but for rotating magnetic perturbations with frequency $f=10^{4} \mathrm{~Hz}$. Curve 1 is for $\sigma=+1 ; 2$ is for $\sigma=-1$.

toroidal drift the ion diffusion coefficients are smaller than $D_{m}$ for co-passing particles and larger than $D_{m}$ for counterpassing particles.

The rotating magnetic field perturbation increases the diffusion rate for co-passing particles due to an outward shift of resonant drift surfaces (curve 1), and decreases it for counterpassing particles due to inward shifted resonant drift surfaces (curve 2) [see Fig. 6(b)]. For instance, at $r$ $=44.2 \mathrm{~cm} D_{i}$ is increased from the value $0.39 \mathrm{~m}^{2} / \mathrm{s}$ in the static case to $0.56 \mathrm{~m}^{2} / \mathrm{s}$ in the rotating case for co-passing particles $\sigma=+1$, and it is, respectively, decreased from $0.57 \mathrm{~m}^{2} / \mathrm{s}$ to $0.43 \mathrm{~m}^{2} / \mathrm{s}$ for the counterpassing particles.

A description of electron and particle transport by diffusion coefficients fails in the so-called laminar zone, i.e., the region of the ergodic zone close to the divertor coils where a majority of field lines have short wall to wall connection lengths. The transport of heat and particles in this region is of convective nature as was discussed in Ref. 42.

\section{CONCLUSIONS}

In this paper, we have studied the effect of external resonant magnetic perturbations on the test particle drift motion in a toroidal system. The study was based on an analysis of Hamiltonian guiding-center equations proposed in Ref. 31. In the case of static magnetic perturbations, the problem is 
reduced to the dynamics of an autonomous two-dimensional Hamiltonian system. The effect of a rotating magnetic field is equivalent to the action of a time-periodic perturbation. For passing particles, the equations for the guiding-center motion were also formulated in Hamiltonian form with the toroidal angle as an independent time-like variable. Field line equations can be directly obtained from these equations. The formulation is suitable to study the deviation of drift orbits from the magnetic field lines. Since it is difficult to obtain analytical expressions for the Hamiltonian in action-angle variables, the Hamiltonian has been represented numerically as a Fourier series in angle variables, with cubic spline interpolations of its harmonics in action variables. It allowed one to integrate the Hamiltonian guiding-center equations using fastrunning symplectic mapping methods developed in Refs. 16, 17 , and 19.

We have analyzed the onset of chaotic drift motion due to resonant magnetic field perturbations. The chaotic region at the plasma edge is formed due to interactions of several resonant drift islands. Their radial positions with respect to the divertor coils, and the distances between them, are important factors in formation of particle chaos. These factors are determined not only by the plasma parameters, as in the case of magnetic field line stochasticity, but also by the particle parameters. It was shown that due to a toroidal drift the positions of resonant drift surfaces are shifted with respect to resonant magnetic surfaces. The shift depends not only on the energy of particles but also on their directions of motion. For co-passing particles the resonant surfaces are shifted inwardly, while for counterpassing particles outwardly. An equilibrium electric field and a rotating magnetic field also significantly vary the positions of resonant drift surfaces, acting selectively on the co-passing and counterpassing particles, respectively. For instance, a rotating field of frequency $f=10^{4} \mathrm{~Hz}$ shifts the resonant drift surface outward (inward) up to $2-3 \mathrm{~cm}$ for the co-passing (counterpassing) particles. The shift of resonant drift surfaces significantly affects the formation of the stochastic zone of drift motion since the magnetic field perturbation strongly decays in the inward radial direction. Particularly, it has been shown that the ergodic regions for co-passing and counterpassing particles are different. This difference becomes more pronounced with an increase in particle energy.

The effects of finite ion gyroradii on the ion transport in the stochastic magnetic field of the TEXTOR-DED are insignificant. This is because that the characteristic radial and poloidal scales of the perturbation field are much larger than the gyroradius of thermal ions. Indeed, the characteristic length $L_{r}=B_{r}\left(d B_{r} / d r\right)^{-1}$ of radial variation of the perturbation field is of order of $L \approx r / m_{0} \sim 2-3 \mathrm{~cm}$ at the plasma edge, while the gyroradius of thermal ions $\left(E_{i}\right.$ $=50-100 \mathrm{eV})$ is $0.3-0.5 \mathrm{~mm}$.

Radial transport of test particles in the stochastic zone can be analyzed by calculating the radial diffusion coefficients. It has been shown that the ion diffusion coefficient has the order of magnitude of the observed anomalous perpendicular diffusion. The diffusion coefficient has different values for co-passing and counterpassing particles. The electron diffusion coefficients are larger than the ion diffusion rate by the factor $\sqrt{m_{i} / m_{e}}$. Because of their small Larmor radii, they are mainly determined by the magnetic field line diffusivity.

In the present study we have not taken into account some important issues of particle transport in fusion devices. First, we did not take into account particle collisions, and second we ignored electrostatic fluctuations which are believed to be also responsible for anomalous (perpendicular) transport of heat and particles. These problems, which are important in a transport study of thermal particles in stochastic magnetic fields, require separate investigations.

\section{ACKNOWLEDGMENT}

This work has been performed under the auspices of the SFB 591.

\section{APPENDIX A: MODELS FOR THE TOKAMAK MAGNETIC EQUILIBRIUM AND MAGNETIC PERTURBATIONS}

We consider the tokamak plasma with nested, circular magnetic surfaces. For a low $\beta$ and large aspect-ratio tokamak plasma, the equilibrium field is described by the vector potential (see Ref. 35, p. 32)

$$
\begin{aligned}
& \mathbf{A}=\left(0, A_{\varphi}(R, Z), A_{z}(R, Z)\right), \\
& A_{\varphi}(R, z)=\frac{B_{0}}{R} \int \frac{d \Psi}{q(\rho(\Psi))}, \\
& A_{z}(R)=-B_{0} R_{0} \ln \left(R / R_{0}\right),
\end{aligned}
$$

where $q(\rho)$ is a safety factor. The quantity

$$
\Psi=R_{p}^{2}(\rho)\left[1-\left(1-\frac{\rho^{2}}{R_{p}^{2}(\rho)}\right)^{1 / 2}\right] \approx \frac{\rho^{2}}{2}
$$

is proportional to the toroidal magnetic flux through a magnetic surface of radius $\rho ; R_{p}(\rho)$ is the major radius of its center. We will use the following model for the Shafranov shift $\Delta(\rho)$ considered in Ref. 49:

$$
\begin{aligned}
\Delta(\rho) & =\left[R_{p}^{2}(a)+(\Lambda+1)\left(a^{2}-\rho^{2}\right)\right]^{1 / 2}-R_{p}(a) \\
& \approx(\Lambda+1) \frac{a^{2}-\rho^{2}}{2 R_{p}(a)},
\end{aligned}
$$

where $\Lambda=\beta_{\mathrm{pol}}+l_{i} / 2-1, l_{i}$ is the internal inductance. The relation between the radius $\rho$ of the magnetic surface and the cylindrical coordinates $R, Z$ is $\rho=\sqrt{\left(R-R_{p}(a)-\Delta(\rho)\right)^{2}+Z^{2}}$.

Consider a model of magnetic perturbations in the DED of the TEXTOR (see Refs. 35 and 45). It consists of 16 helical conductors on inboard circumference of radius $r_{c}$ aligned parallel to the magnetic field lines. They are located in the poloidal sector $\pi-\theta_{c}<\theta<\pi+\theta_{c}$ on the high field side (HFS) with the width $\Delta \theta=2 \theta_{c}$. In the standard operational mode the current distribution over coils is given by $I_{j}$ $=I_{d} \sin (\pi j / 2+\Omega t), j=1, \ldots, 16$, which creates resonant magnetic perturbations $m: n$ with the toroidal mode $n=4$ and the several poloidal modes near the magnetic surface $q=3$. The current amplitude $I_{d}$ may reach up to $15 \mathrm{kA}$, and rotation 
frequencies up to $\Omega=10^{4} \mathrm{~Hz}$. The magnetic perturbation created by the coil system may be well described by the vector potential $\mathbf{A}=\left(0, A_{\varphi}, 0\right)$ (see Ref. 35 , p. 22):

$$
A_{\varphi}(r, \theta, \varphi)=\sum_{m} A_{m}(r) \cos (m \theta-n \varphi-\Omega t),
$$

where

$$
A_{m}(r) \approx-\frac{B_{c} g_{m}}{m} r_{c} \sqrt{\frac{R_{0}}{R}}\left(\frac{r}{r_{c}}\right)^{|m|} .
$$

Here $B_{c}=\mu_{o} I_{d} n \cos \alpha_{0} / \theta_{c} r_{c}$ is a characteristic amplitude of magnetic perturbations with $\alpha_{0}=\theta_{c} r_{c} / \pi\left(R_{0}-r_{c}\right)$. The quantity

$$
g_{m}=(-1)^{m} \frac{\sin \left[\left(m-m_{0}\right) \theta_{c}\right]}{\pi\left(m-m_{0}\right)}
$$

describes the spectrum of poloidal modes. In the standard case the central mode $m_{0} \approx 20$. For the numerical calculations we have chosen the divertor current $I_{d}=15 \mathrm{kA}$, minor coil radius $r_{c}=53.25 \mathrm{~cm}$, and half poloidal extension $\theta_{c}$ $=\pi / 5$.

\section{APPENDIX B: DRIFT HAMILTONIAN IN ACTION-ANGLE VARIABLES}

It is convenient to describe the drift motion of confined particles in action-angle variables $I_{z}, I_{\varphi}, \vartheta_{z}, \vartheta_{\varphi}$. The action variables $I_{z}, I_{\varphi}$ follow from the unperturbed Hamiltonian $H_{0}$ according to

$$
\begin{aligned}
& I_{z}=\frac{1}{2 \pi} \oint_{C_{p}} P_{z}\left(Z ; P_{\varphi}, H_{0}\right) d Z, \\
& I_{\varphi}=\frac{1}{2 \pi} \oint_{C_{\varphi}} P_{\varphi} d \Phi,
\end{aligned}
$$

where the integrals is taken along the projections $C_{p}$ and $C_{\varphi}$ of drift orbit onto the poloidal $\left(x, P_{z}\right)$ and the toroidal $\left(x, P_{\varphi}\right)$ sections of the phase space, respectively. In action variables $H_{0}=H_{0}\left(I_{z}, I_{\varphi}\right)$. The change of the old variables $Z, \Phi, P_{z}, P_{\varphi}$ to the new action-angle variables $I_{z}, I_{\varphi}, \vartheta_{z}, \vartheta_{\varphi}$ follows from the generating function

$$
S=S\left(Z, \Phi, I_{z}, I_{\varphi}\right)=\Phi I_{\varphi}+\int^{Z} P_{z}\left(Z^{\prime} ; I_{z}, I_{\varphi}\right) d Z^{\prime} .
$$

Then the relations between the old variables and the new angle variables are

$$
\begin{aligned}
& \vartheta_{z}=\frac{\partial}{\partial I_{z}} \int^{Z} P_{z}\left(Z^{\prime}, I_{z}, I_{\varphi}\right) d Z^{\prime}, \\
& \vartheta_{\varphi}=\Phi+\frac{\partial}{\partial I_{\varphi}} \int^{Z} P_{z}\left(Z^{\prime}, I_{z}, I_{\varphi}\right) d Z^{\prime} .
\end{aligned}
$$

The coordinates $Z, \Phi$ are periodic functions of the angle variables $\vartheta_{z}, \vartheta_{\varphi}$ :

$$
Z=Z\left(\vartheta_{z}, \vartheta_{\varphi} ; I_{z}, I_{\varphi}\right)=\sum_{m, n} z_{m}\left(I_{z}, I_{\varphi}\right) \exp \left(i m \vartheta_{z}\right),
$$

$$
\Phi=\Phi\left(\vartheta_{z}, \vartheta_{\varphi} ; I_{z}, I_{\varphi}\right)=\vartheta_{\varphi}+\sum_{m, n} \Phi_{m}\left(I_{z}, I_{\varphi}\right) \exp \left(i m \vartheta_{z}\right)
$$

The unperturbed motion is characterized by the poloidal, $\omega_{z}\left(I_{z}, I_{\varphi}\right)=\partial H_{0} / \partial I_{z}$, and the toroidal, $\omega_{\varphi}\left(I_{z}, I_{\varphi}\right)=\partial H_{0} / \partial I_{\varphi}$, transit frequencies of the conditionally periodic motion on the drift surface $H=H_{0}\left(I_{z}, I_{\varphi}\right)=H_{0}\left(Z, P_{z}, I_{\varphi}\right)=$ const. The angle variables $\vartheta_{z}$ and $\vartheta_{\varphi}$, determining the position of the guiding center on the drift surface, are linear functions of time: $\vartheta_{z}=\omega_{z}\left(I_{z}, I_{\varphi}\right) t+\vartheta_{z 0}, \vartheta_{\varphi}=\omega_{\varphi}\left(I_{z}, I_{\varphi}\right) t+\vartheta_{\varphi 0}$.

In action-angle variables, the total Hamiltonian takes the form

$$
H=H_{0}\left(I_{z}, I_{\varphi}\right)+\epsilon H_{1}\left(\vartheta_{z}, \vartheta_{\varphi}, I_{z}, I_{\varphi}, t\right),
$$

where the perturbed Hamiltonian $\epsilon H_{1}$ can be presented as a Fourier series in $\vartheta_{z}, \vartheta_{\varphi}$ :

$$
\epsilon H_{1}=\epsilon \sum_{m, n} H_{m n}\left(I_{z}, I_{\varphi}\right) \cos \left(m \vartheta_{z}-n \vartheta_{\varphi}+\Omega t\right) .
$$

The spectral components, $H_{m n}\left(I_{z}, I_{\varphi}\right)$, of the perturbed Hamiltonian $H_{1}$ are determined by the Fourier integrals:

$$
\begin{aligned}
H_{m n}\left(I_{z}, I_{\varphi}\right)= & \frac{\Omega}{8 \pi^{3}} \int_{0}^{2 \pi} d \vartheta_{z} \int_{0}^{2 \pi} d \vartheta_{\varphi} \int_{0}^{2 \pi / \Omega} d t \\
& \times H_{1}\left(Z\left(\vartheta_{z}\right), \Phi\left(\vartheta_{z}, \vartheta_{\varphi}\right), P_{z}\left(\vartheta_{z}\right), P_{\varphi}, t\right) \\
& \times e^{-i m \vartheta_{z}+i n \vartheta_{\varphi}-i \Omega t} .
\end{aligned}
$$

The drift motion is governed by

$$
\begin{aligned}
& \frac{d \vartheta_{\alpha}}{d t}=\frac{\partial H}{\partial I_{\alpha}}=\omega_{\alpha}\left(I_{z}, I_{\varphi}\right)+\epsilon \frac{\partial H_{1}}{\partial I_{\alpha}}, \\
& \frac{d I_{\alpha}}{d t}=-\epsilon \frac{\partial H_{1}}{\partial \vartheta_{\alpha}}, \quad \alpha=z, \varphi .
\end{aligned}
$$

According to the Kolmogorov-Arnold-Moser (KAM) theory most drift surfaces may survive under the perturbation (B9) if the perturbation is sufficiently small and far from the resonant drift surfaces. The latter are determined by the condition

$$
m \omega_{z}\left(I_{z}, I_{\varphi}\right)-n \omega_{\varphi}\left(I_{z}, I_{\varphi}\right)+\Omega=0,
$$

with certain integer numbers $m, n$. The resonant drift surfaces are significantly affected by the perturbation. Consider the drift orbits near the $(m, n)$ resonance surface. Following Ref. 14 we introduce the new canonical phases $\psi, \psi_{\varphi}$ and the actions $J, J_{\varphi}$, with the help of the generating function $F$ $=\left(m \vartheta_{z}-n \vartheta_{\varphi}+\Omega t\right) J+\vartheta_{\varphi} J_{\varphi}$. Then, taking into account only the dominating single resonance perturbation $H_{1}$ $=\epsilon H_{m n} \cos \left(m \vartheta_{z}-n \vartheta_{\varphi}+\Omega t\right)$ the canonical equations can be transformed to new ones with the new Hamiltonian function

$$
\begin{aligned}
H_{J}\left(\psi, J, J_{\varphi}\right)= & H_{0}\left(m J,-n J+J_{\varphi}\right)+\Omega J \\
& +\epsilon H_{m n}\left(m J,-n J+J_{\varphi}\right) \cos \psi .
\end{aligned}
$$

The relations between the old action variables $I_{z}, I_{\varphi}$, and the new one $J$ are 


$$
\begin{aligned}
& \psi=\frac{\partial F}{\partial J}=m \vartheta_{z}-n \vartheta_{\varphi}+\Omega t, \quad \psi_{\varphi}=\frac{\partial F}{\partial J_{\varphi}}=\vartheta_{\varphi}, \\
& I_{z}=\frac{\partial F}{\partial \vartheta_{z}}=m J, \quad I_{\varphi}=\frac{\partial F}{\partial \vartheta_{\varphi}}=-n J+J_{\varphi} .
\end{aligned}
$$

From (B14) it follows that $n I_{z}+m I_{\varphi}=J_{\varphi}$ is an integral of motion. Suppose that the resonance condition (B13) is satisfied for $J=J_{m n}$. The canonical equations for the new variables $\left(\psi, \psi_{\varphi}, J, J_{\varphi}\right)$ can be simplified by expanding the Hamiltonian (B14) in series of powers of $\Delta J=J-J_{m n}$, and neglecting the terms proportional to $\epsilon \Delta J$. Then we obtain

$$
\frac{d \psi}{d t}=\frac{\partial H_{J}}{\partial J}=\kappa_{m n} \Delta J, \quad \frac{d \Delta J}{d t}=-\frac{\partial H_{J}}{\partial \psi}=\epsilon V_{m n} \sin \psi,
$$

$$
H_{J}=\frac{\kappa_{m n}}{2}(\Delta J)^{2}+\epsilon V_{m n} \cos \psi
$$

where $V_{m n} \equiv H_{m n}\left(m J_{m n},-n J_{m n}+J_{\varphi}\right)$ and

$$
\kappa_{m n}=\left(m \frac{\partial}{\partial I_{z}}-n \frac{\partial}{\partial I_{\varphi}}\right) \tilde{\Omega} \text {. }
$$

Here, $\widetilde{\Omega}=m \omega_{z}-n \omega_{\varphi}$. Equations (B17) correspond to a pendulum with a frequency (for small amplitude oscillations) $\nu_{m n}=\sqrt{\left|\epsilon V_{m n}\right| / \kappa_{m n}}$. For $-\kappa_{m n} \nu_{m n}^{2}<H_{J}<\kappa_{m n} \nu_{m n}^{2}$ the particle orbits are trapped by the chain of drift islands. The widths of the islands, $W_{m n}=2 \max |\Delta J|$, are

$$
W_{m n}=4 \nu_{m n}=4 \sqrt{\left|\epsilon V_{m n}\right| / \kappa_{m n}} .
$$

In the old action variables $I_{z}, I_{\varphi}$ we have $\delta I_{z}=m W_{m n}$ and $\delta I_{\varphi}=n W_{m n}$. Taking into account that near the resonance the variables are not independent, one can easily show that the quantity $\kappa$ can be written as $\kappa_{m n}=\partial \widetilde{\Omega} / \partial \bar{J}$, where $\bar{J}=I_{z} / m$ $-I_{\varphi} / n=2 I_{z} / m-J_{\varphi}$.

${ }^{1}$ R. Balescu, Transport Processes in Plasmas: 2. Neoclassical Transport Theory (North-Holland, Amsterdam, 1988).

${ }^{2}$ A. A. Galeev and R. Z. Sagdeev, in Handbook of Plasma Physics, Basic Plasma Physics I, edited by A. A. Galeev and R. N. Sudan (NorthHolland, Amsterdam, 1984), p. 679.

${ }^{3}$ R. D. Hazeltine and J. D. Meiss, Plasma Confinement (Addison-Wesley, Redwood City, 1992).

${ }^{4}$ R. Balescu, Aspects of Anomalous Transport in Plasmas (Institute of Physics, Bristol, 2005).

${ }^{5}$ R. Balescu, Statistical Dynamics, Matter Out of Equilibrium (Imperial College, London, 2000).

${ }^{6}$ A. H. Boozer and R. B. White, Phys. Rev. Lett. 49, 786 (1982).

${ }^{7}$ R. B. White and Y. Wu, Plasma Phys. Controlled Fusion 35, 595 (1993).
${ }^{8}$ H. E. Mynick, Phys. Fluids B 5, 1471 (1993).

${ }^{9}$ H. E. Mynick, Phys. Fluids B 5, 2460 (1993).

${ }^{10}$ R. B. White, Phys. Rev. E 58, 1774 (1998).

${ }^{11}$ M. DeRover, A. M. Schilham, A. Montvai, and N. J. Lopes Cardozo, Phys. Plasmas 6, 2443 (1999).

${ }^{12}$ T. E. Evans, R. A. Moyer, P. R. Thomas, J. G. Watkins, T. H. Osborne, J. A. Boedo, E. J. Doyle, M. E. Fenstermacher, K. H. Finken, R. J. Groebner et al., Phys. Rev. Lett. 92, 235003 (2004).

${ }^{13}$ H. E. Mynick and N. Pomphrey, Nucl. Fusion 34, 1277 (1994).

${ }^{14}$ D. D. Pritchard, R. F. Gandy, J. D. Hanson, S. F. Knowlton, H. Lin, G. E. Sasser, E. Thomas, and J. Cooney, Phys. Plasmas 4, 162 (1997).

${ }^{15}$ A. A. Shiskin, Nucl. Fusion 42, 344 (2002).

${ }^{16}$ S. S. Abdullaev, J. Phys. A 32, 2745 (1999).

${ }^{17}$ S. S. Abdullaev, J. Phys. A 35, 2811 (2002).

${ }^{18}$ S. S. Abdullaev, Nucl. Fusion Suppl. 44, S12 (2004).

${ }^{19} \mathrm{~S}$. Abdullaev, Construction of Mappings for Hamiltonian Systems and Their Applications (Springer-Verlag, Berlin, 2006).

${ }^{20}$ S. S. Abdullaev, K. H. Finken, A. Kaleck, and K. H. Spatschek, Phys. Plasmas 5, 196 (1998).

${ }^{21}$ R. Balescu, M. Vlad, and F. Spineanu, Phys. Rev. E 58, 951 (1998).

${ }^{22}$ H. Ali, A. Punjabi, A. Boozer, and T. Evans, Phys. Plasmas 11, 1908 (2004).

${ }^{23}$ A. Punjabi, A. Verma, and A. Boozer, J. Plasma Phys. 52, 91 (1994).

${ }^{24}$ O. Fischer, W. A. Cooper, and L. Villard, Nucl. Fusion 40, 1453 (2000).

${ }^{25}$ R. B. White, A. H. Boozer, and R. Hay, Phys. Fluids 25, 575 (1982).

${ }^{26}$ A. H. Boozer, Phys. Fluids 27, 2441 (1984).

${ }^{27}$ R. B. White and M. S. Chance, Phys. Fluids 27, 2455 (1984).

${ }^{28}$ R. B. White, Phys. Fluids B 2, 845 (1990).

${ }^{29}$ J. D. Meiss and R. D. Hazeltine, Phys. Fluids B 2, 2563 (1990).

${ }^{30}$ H. Wobig and D. Pfirsch, Plasma Phys. Controlled Fusion 43, 695 (2001).

${ }^{31}$ S. S. Abdullaev and K. H. Finken, Phys. Plasmas 9, 4193 (2002).

${ }^{32}$ H. E. Mynick, Phys. Fluids 23, 1888 (1980).

${ }^{33}$ H. E. Mynick, Phys. Fluids 23, 1897 (1980).

${ }^{34}$ K. H. Finken, Fusion Eng. Des. 37, 335 (1997).

${ }^{35}$ K. H. Finken, S. S. Abdullaev, M. Jakubowski, M. Lehnen, A. Nicolai, and K. H. Spatschek, The Structure of Magnetic Field in the TEXTOR$D E D$, Series in Energy Technology (Forschungszentrum Julich, Julich, 2005), Vol. 45

${ }^{36}$ W. Engelhardt and W. Feneberg, J. Nucl. Mater. 76-77, 556 (1978).

${ }^{37}$ W. Feneberg and G. Wolf, Nucl. Fusion 21, 669 (1981).

${ }^{38}$ J. S. deGrassie, N. Ohyabu, N. H. Brooks, K. Gentle, R. Bengston, R. Bravenec, W. Hodge, T. Kochanski, K. Nelin, P. Phillips et al., J. Nucl. Mater. 128-129, 266 (1984).

${ }^{39}$ A. Samain, A. Grossman, T. Blenski, G. Fuchs, and B. Steffen, J. Nucl. Mater. 395, 128 (1984).

${ }^{40}$ A. Wootton, S. C. McCool, and S. Zheng, Fusion Technol. 19, 473 (1991).

${ }^{41}$ P. Ghendrih, A. Grossman, and H. Cape, Plasma Phys. Controlled Fusion 38, 1653 (1996)

${ }^{42}$ A. Grossman, Plasma Phys. Controlled Fusion 41, A185 (1999).

${ }^{43}$ K. H. Finken, S. S. Abdullaev, T. Eich, D. W. Faulconer, M. Kobayashi, R. Koch, G. Mank, and A. Rogister, Nucl. Fusion 41, 503 (2001).

${ }^{44}$ A. Kaleck, M. Hassler, and T. Evans, Fusion Eng. Des. 37, 353 (1997).

${ }^{45}$ S. S. Abdullaev, K. H. Finken, and K. H. Spatschek, Phys. Plasmas 6, 153 (1999).

${ }^{46}$ K. H. Finken, S. S. Abdullaev, A. Kaleck, and G. Wolf, Nucl. Fusion 39, 637 (1999).

${ }^{47}$ S. S. Abdullaev, T. Eich, and K. H. Finken, Phys. Plasmas 8, 2739 (2001).

${ }^{48}$ J. Wesson, Tokamaks (Clarendon, Oxford, 2004).

${ }^{49}$ F. Nguyen, P. Ghendrih, and A. Samain, EUR-CEA-FC (Association Euratom-CEA/Cadarache, 1995). 